\title{
Energy Homeostasis Principle: A Naturalistic Approach to Explain the Emergence of Behavior
}

Sergio Vicencio ${ }^{1}$, Mario Villalobos ${ }^{2}$, Pedro Maldonado ${ }^{3}$, Rodrigo C. Vergara ${ }^{4}$

1 The Center for Hearing and Balance, Otolaryngology-Head and Neck Surgery, Johns Hopkins University School of Medicine, Baltimore, MD.

${ }^{2}$ Escuela de Psicología y Filosofía, Universidad de Tarapacá, Arica, Chile.

${ }^{3}$ Laboratorio de Neurosistemas, Departamento de Neurociencia \& BNI, Facultad de Medicina, Universidad de Chile, Santiago, Chile

4 Departamento de Kinesiología, Facultad de Artes y Educación Física, Universidad Metropolitana de las Ciencias de la Educación.

*Correspondence:

Rodrigo C. Vergara. Ph.D.

Departamento de Kinesiología

Facultad de Artes y Educación Física

Dr. Luis Bisquert 2765, Ñuñoa, Región Metropolitana

Santiago, Chile.

rodrigo.vergara o@umce.cl

Keywords: Energy, Homeostasis, Neural Network, Behavior, Free Energy Principle 


\section{Abstract}

Explaining the emergence of behavior and understanding on the basis of neuronal mechanisms is still elusive. One renowned proposal is the Free Energy Principle (FEP), which uses an informationtheoretic framework derived from thermodynamic considerations to describe how behavior and understanding would emerge. FEP starts from a whole organism approach, based on mental states and phenomena, mapping them into the neuronal substrate. An alternative approach, the Energy Homeostasis Principle (EHP), initiates a similar explanatory effort, but starting from single neuron phenomena and building up to the whole organism's behavior and understanding. In this work, we develop the EHP as an alternative but complementary vision to FEP and try to explain how behavior and understanding would emerge from the local requirements of the neurons. Based on EHP and a strict naturalist approach that sees living beings as physical and deterministic systems, we explain scenarios where learning would emerge without the need for volition or goals. Given these starting points, we state several considerations of how we see the nervous system, particularly the role of function, purpose, and the conception of goal-oriented behaviors. We problematize these conceptions, giving an alternative teleology-free framework in which behavior and, ultimately, understanding would still emerge. We reinterpret neural processing explaining basic learning situations up to simple anticipatory behavior. Finally, we end the work with an evolutionary perspective of how this non-goal-oriented behavior appears. We acknowledge that in the current form of our proposal, we are still far from explaining the emergence of understanding. Still, we set the ground for an alternative neuron-based framework to ultimately explain understanding. 


\section{Introduction}

When an animal displays different behaviors, what are the primary processes occurring in the nervous system? How do neurons, neuronal networks, and ultimately the whole nervous system participate in behavior generation? This paper argues that the nervous system unfolds autogenous mechanisms of energetic homeostasis, maintaining its energy equilibrium as a system. In our view, the nervous system operates in the continuous present tense of its structural dynamics under strictly local rules of energy stability, without pursuing biological goals or adaptive adjustments for the organism. This spontaneous process of maintaining its energy balance occurs so that under statistically normal anatomical, physiological, and ecological conditions, it results precisely in those behaviors that prove to be adaptive for the animal.

This view of the nervous system corresponds, in essence, to what has been recently introduced as the Energy Homeostasis Principle (EHP) (Vergara et al., 2019). This theoretical proposal draws strongly from the autopoietic theory of cognition in the sense of being strictly naturalistic (Maturana, 1978; Villalobos, 2015; Villalobos and Ward, 2015), and resonates, although with important nuances, with some aspects of the FEP approach in theoretical neuroscience (Friston, 2010; Friston and Stephan, 2007). The EHP, as we will see, does not hold that animal behavior and cognition arise just because the nervous system is a homeostatic energy system. If that were the case, we should observe cognition and complex behavior in any homeostatic energy system, as may occur in an open thermodynamic system that exhibits some degree of stability, such as tornadoes and stars (McGregor and Virgo, 2011; Ulanowicz and Hannon, 1987). Instead, the proposal is to realize that while we observe the behaviors or cognition manifested by an organism, the way its nervous system operates is simply obeying, in its way, the EHP. The nervous system is a homeostatic energy system, like other similar natural systems, but with significant structural and organizational features that 
make it unique in its way. These structural and organizational features are essential because they are the ones that explain why the nervous system, despite operating under the EHP, can generate phenomena such as animal behavior and cognition. The argument EHP wants to make is that despite all the unique features we may find in the nervous system, it remains the fact that its operations obey, ultimately, homeostatic energy mechanisms.

This latter statement merits further discussion. When we speak of the unique features in the nervous system, we are not inviting the reader to picture mysterious non-natural features. All thermodynamic systems that maintain stability and integrity for the period they exist, long or short, have their own features related to their specific structural compositions and dynamic patterns. Candle flames and tornadoes are both dissipative structures that exhibit thermodynamic stability in their scales. Still, only candle flames generate exothermic fast combustion reactions, radiate light, and illuminate a dark room. Conversely, tornadoes, not candle flames, can travel kilometers through large geographic areas, lifting and violently shaking heavy objects. There is nothing mysterious about these differences. They relate to each system's respective chemical and physical features, and they are the ones that must be considered if we want to explain the different phenomena associated with each system. What is it doing as a system when the candle flame radiates light and warms up our hands? From the systemic thermodynamic point of view, it is simply maintaining its stability and integrity as a dissipative system. When the tornado passes through the village and destroys the houses, what is it doing as a system? Again, from the systemic thermodynamic point of view, it is simply maintaining its stability and integrity as a dissipative system. But, if both systems are just doing the same thing, why do they generate such different phenomena and results? The answer lies in the unique features of each system, the context in which they form, their material qualities, and so on. 
The nervous system is a homeostatic energy system. Still, the specific way it unfolds such a condition, given its biological (e.g., histological) composition, its anatomical structure and physiological organization, its looping coupling with both the internal milieu and the external environment, its formation, and development within the organism, generates results and phenomena that are distinctive and that we call behavior and cognition. In what follows, we will expose the general systemic conditions that run for the nervous system.

\section{General systemic conditions}

To understand the nervous system and the phenomena typically associated with its functioning (e.g., perception, motor control, language, consciousness), it is crucial to attend to its peculiarities and distinctive features as a system. However, it is equally important to consider those conditions that make the nervous system a natural system among all natural systems, living or not. That is, to the conditions that the nervous system shares with the rest of natural systems, and according to which it must work. After all, what is fascinating about the nervous system is that being a natural system, that is, a system that must respect the laws, conditions, and principles that rule and restrict every natural system, it can generate phenomena as peculiar and exceptional as perceptual experience, understanding, consciousness, language, and intelligent reasoning.

This latter explanatory exercise is essential because, when facing extremely complex explanatory problems, it is usually tempting and easy to recur to the strategy of endowing the components and explanatory machinery of the system under study with the very special and complex properties we want to explain. For instance, this was the case with the explanation of the phenomenon of life. For an extended period, it was assumed that the components of the living beings were unique in that they were endowed with a certain kind of vital force or energy that was not present in the 
components of inert objects (Bechtel and Richardson, 1998). We tried to explain life by postulating that the matter of which living beings are made was itself, somehow, living. Similarly, when facing the problem of explaining cognitive and mental phenomena, such as perception or intelligent reasoning, it is tempting to think of the nervous system, its components, and machinery, as if they operated themselves with proto (subpersonal, automatic, unconscious) cognitive mechanisms. As if the nervous systems were itself a kind of epistemic agent dealing with alleged problems of uncertainty and lack of information, working on the base of hypotheses, inferences, predictions, error detection, and looking for evidence and hypothesis confirmation.

As the case of biology and the problem of life teach us, the strategy of projecting the properties and capacities of the explanandum, even in a carefully sophisticated deflationary way, into the explanatory substratum itself does not lead to adequate explanations. We think we do better if we take the nervous system not as a cognitive agent but as a physical machine (Ashby, 1947) and try to understand its functioning according to the conditions that rule for every physical system in general. Doing this does not mean, of course, ignoring the particular features of the nervous system regarding its structure and organization; it just means understanding that such specific features do not set the nervous system apart from the rest of the natural systems.

Before we further develop our argument for a strict naturalistic approach to explain the emergence of behavior, we consider it essential to lay out some foundational concepts, so the reader can better consider the starting points. These points are not meant to provide an exhaustive characterization of the nervous system; far from that. However, combined, they should help us to understand, in broad terms, the way the nervous system operates and generates some of the phenomena associated with its functioning. We consider the following premises: 
1. The nervous system is non-teleological. Its dynamics are not driven by purposes or goals. As is the case with natural systems in general, the dynamics of the nervous system unfold obeying physical laws that are blind to purposes or goals (Villalobos and Ward, 2015).

2. The nervous system is non-normative. Its dynamics are not based on normative considerations such as what is (or might be) good or bad, adequate, or inadequate, beneficial, or harmful for the system itself or the organism. As is the case with natural systems in general, the dynamics of the nervous system unfold obeying physical laws that are blind to normative values (Villalobos and Ward, 2015).

3. The interactions of the nervous system with its surrounding systems, both intra- and extraorganism, are structural (i.e., physical, chemical, energetic) in nature, not epistemic, informative, or cognitive (Maturana, 2002). The nervous system is not an epistemic agent that gets and processes information, and its functioning is not oriented to knowing (inferring, predicting, guessing) anything (Villalobos, 2015).

4. The components of the nervous system, its neurons, and networks work through strictly local interactions, without "having in view" distal states, either intra- or extra-organism (Maturana and Varela, 1989).

5. The nervous system operates in its continuous structural present, without "having in view" noncurrent states, either past or future (Ashby, 1960; Maturana, 2008).

6. The nervous system, at the neuroscience scale of analysis, behaves deterministically (Ashby, 1960; Maturana and Varela, 1980). It is not a free agent that chooses, among a set of possibilities, what to do. The nervous system does what it does at every instant because its structure at that instant simply allows no other action. 
7. The nervous system is an open thermodynamic system that exchanges matter and energy with its surrounding.

8. The nervous system is a homeostatic system that, like all homeostatic systems, maintains certain stability and equilibrium in its physical parameters and shows the capacity to restore them when they are disturbed within specific ranges (Ashby, 1960).

9. Nervous systems, since their first formation in the embryonal stage, grow and develop in the continuous coupling, adaptation, and structural coherence with their biological surrounding and the extra-organism environment. This is a trivial condition for every system. Everything that arises and begins to exist does so because the conditions for its emergence and existence are given. Every system is born adapted to, or in structural coherence with, its surrounding conditions. This adaptation is conserved while the system exists as such and lost when the system ceases to exist.

10. A nervous system with normal anatomical and physiological development is always coupled in a loop with (i) other physiological systems of the organism, such as the endocrine, immune, cardiovascular and digestive systems, and (ii) the external environment through specialized sensory organs and motor structures. Since these couplings are functionally closed as feedback loops, the nervous system always affects itself through them and thus maintains its homeostasis. At the same time, since these couplings, from the beginning, arise in structural coherence and adaptation (recall point 9), the self-centered homeostatic dynamics of the nervous system result in the conservation of the adaptation of the rest of the organism.

During the next sections, we will elaborate on the EHP considering this set of premises to produce a plausible explanation for behavior and ultimately understanding. We will start arguing how a naturalistic approach is required to disentangle proximate causes (cell operation) from distal causes (organism operations). Then we will build over such conception to reinterpret neural processing 
without goal and purpose. We will also evaluate anticipatory behavior by means of EHP and contrasting it with FEP. Finally, we will expose an evolutionary argument of how these apparently goal-directed-behavior emerge from non-teleological mechanisms. Even more, we will discuss how useless behavior may appear and it may constitute a potential adaptive advantage in evolutionary terms.

\section{Some specific considerations about the nervous system}

One way to illustrate how neurons interactions are restricted, and therefore, locally driven interactions dynamics, is to realize their context. When comparing the whole organism with its component cells, or even organs, it can be noted that they are sensitive to completely different scales of physical phenomena (Dada and Mendes, 2011; Southern et al., 2008). For instance, swimming in a pool or the ocean makes little difference to an experienced swimmer, whereas doing so in an aqueous solution would be lethal to a cell (Pedersen et al., 2011). This becomes very clear at the spatial and temporal scales (Dada and Mendes, 2011; DiFrisco, 2017; Engel, 1980; Southern et al., 2008). For example, at the chemical level, cells are most sensitive to their direct environment, to a space on the order of micrometers or less. Whereas we, as organisms, are sensitive to millimeters and beyond. In terms of time scale, the difference is equally remarkable. Most of our cells are replaced in our lifetime (DiFrisco, 2017), which means their time scale is significantly smaller than ours.

We may argue the specifics of these differences. Up to which point the scales are overlapped, or how arbitrary is even to state that such scales even exist. However, the core of that observation goes beyond the scales themselves, the point is that the phenomenological operational closure of a whole human being compared with a single cell. What I see as a hamburger is not the same 
experience for a cell. On the one hand, a cell is too tiny to perceive the hamburger as a whole, but also the potential interactions with it are different from those we would engage in. There is a difference in how we perceive and the actions we may perform given such perception; how we couple with objects in behavior. As such, even if we would acknowledge that a neuron or neural network would foresee something, it would be in a shorter time span and reading their local interactions.

Given our aim to explain the emergence of behavior from a naturalistic viewpoint, the difference in sensitivity is relevant for at least two reasons. The first reason is the difficulty in establishing relationships between these levels; if they don't perceive the same phenomena, how are their dynamics aligned for survival? This complicates the development of causal explanations in biology. A similar situation was noticed 60 years ago by Ernst Mayr (1961) when he identified that virtually all explanations of biological phenomena consisted of sets of proximate causes and sets of ultimate causes (or distal given our framework). In this work, he illustrates the difficulties in establishing the causes of behaviors, arguing that they can be attributed to the environment, physiology (including molecular mechanisms), or the interaction between the two. In this context, proximate causes would be those that control the organism's responses to immediate environmental factors (such as the sunrise regulation of the sleep-wake cycle in a mouse), while ultimate causes would be those that have an impact on the organism's survival (such as increased nocturnal activity in mice that decreases the probability of encountering predators). These ultimate causes are rooted in evolutionary mechanisms and have been incorporated into the system through generations of natural selection (Mayr, 1961). Therefore, under the EHP view, behavior emerges from the intersection of fulfilled local interactions, which keep cells alive and evolutionary pressure which 
admit local conditions to be fulfilled, if they don't jeopardize the whole organism's life (the distal cause).

There is a second reason; for the organism to survive, the fundamental needs of all these levels must be met. The specific needs of different cell kinds are varied and different from those of the organisms they compose. Therefore, we have multiple layers or levels of operational closure which are not strictly equivalent nor overlapped, which must be fulfilled for the entire organism to keep alive and coupled with its environment. This illustrates the complex synchronization that must occur in such an organism's cell population to survive.

Now, an apparent contradiction appears. Despite the short overlap of sensitivity to phenomena between the parts of our body and the whole organism, we exhibit adaptative behaviors. This supposed paradox has been resolved mainly by assigning functions aimed at the survival of the entire organism to different parts of the body (Roux, 2014). However, this position usually omits the evolutionary process which led to such functions while also neglecting the survival of the cells that live in us. It is critical to note that many of our cells die daily and that each of these cells has different survival requirements and may not act in alignment with the survival of the whole organism. This is evident in pathologies such as cancer (Chaffer and Weinberg, 2011) and autoimmune diseases (Park and Kupper, 2015). We tend to refer to these conditions as errors or problems of specific systems and functions, forgetting that, since cells live in us, but not for us, there is a possibility that these phenomena may occur.

An alternative approach would be to consider that each cell is solving its own requirements to survive. In this sense, it is essential to assume that the cell, as an autopoietic unit, is able to respond and exert control over its niche, but only within its local environment. Thus, specific environmental conditions that occur in localized regions of our body will set in motion different cellular mechanisms. 
Since cells can only directly influence that local environment, they can only solve their requirements. But, when all cells solve their requirements, they, most of the time, indirectly end up solving ours. As such, behavior can be considered an emergent property derived from the individual actions of cells that lead to their survival and, ultimately to ours. These two levels must be aligned for the whole organism to survive; however, there is a possibility of mismatches where some are neutral (without significant consequences) while others give rise to what we call pathologies.

This different approach can be described as an interaction of parallel causes and requirements nested in cells and organisms, in the sense that the phenomena present in individualized cells mirror a distal effect on the whole organism and vice versa. Therefore, we may explain behavior from the entire organism's view or its cells' interactions. However, a comprehensive approach would be to track cells interaction up to the mirrored effect on the organisms. Critically, without neglecting that the proximal causes of each layer or level are aligned for survival. As such, the same phenomena can present a different impact on the organism and cells populations within. For instance, covering the head with the hands to block something that would hit our head is adaptive for the organisms, yet hands cells will die during the event, therefore not being adaptive for them.

At this point, we may start asking which the most relevant aspect of cells' survival is. Naturally, energy managing in any cell is critical for survival, as they must balance expenditure with income, allowing them to have a reserve big enough to cope with environmental restrictions. If we consider a cell that lives within an organism, with an evident impact over organisms' behavior such as a neuron, this premise stands. For neurons to survive, they must properly manage their energy budget. Problems from the organism, such as avoiding injuries, coupling with stressful work, dealing with close one death, and so on, are not part of the proximal phenomena stressing a single neuron. Of course, those phenomena have stimuli transduction into local neural requirements: energy demand. 
Therefore, neurons will deploy mechanisms to couple with their local requirements, and hopefully, they will solve organisms' problems as well. As such, whenever describing how behavior emerges, we should always map the differences between organism and cells' domains of interactions. For instance, Vergara et al. (2019) described perceptual stimuli as mapped into physiology with different impacts at each level. An organism may just be looking at something. At the same time, transduction sets electromagnetic waves of the visual spectrum into action potentials, which in turn produce a cascade effect all over the nervous system, impacting the energetic demands of neurons and glia (Vergara et al., 2019). Depending on how demanding this stimulus energetically is, neurons may regulate their synaptic weights (Barral and Reyes, 2016), producing a new functional network. This new functional network will, in turn, activate muscles leading to visible behavior which may change stimulus (e.g., closing the eyes).

In this framework, the energy balance mechanisms of the cells have a consequential impact on physiology resulting in the emergence of behavior. At the same time, since the cellular level and that of the whole organism are analogous to nested layers or levels, the behavior itself will impact not only the experience of the whole organism but also the cells that compose it. It may be the case that only some of the cells are affected, which remarks the need of recognizing that the same phenomena may impact differently the whole organisms and regions (cells) within. Also relevant, is the fact that neurons cannot directly experience the stimuli which trigger organism behaviors. Once sensorial transduction is made, only proximal phenomena such as action potential, lactate transporter activation, synaptic modulations, and so on are observable. In other words, cells such as our neurons are never solving a mathematical problem, or recognizing a face, but are only solving energetic needs required for their survival. 


\section{Reinterpreting neural processing}

The notion that behavior is not inside the machine is notably exemplified in the experiments in "synthetic psychology" of Braitenberg (Braitenberg, 1986). He presented how simple mechanisms may lead to complex behaviors and the illusion of complex cognitive processing. The complexity may be loaned from the environment, while internal mechanisms can stay simple. We usually think of neural mechanisms as complex and difficult to assess, based on the complexity of behavior. Let us assume for a moment that it might be the case that neural mechanisms are relatively simple and that most of the complexity we see in our behavior is loaned from our environment. Is there an experiment like Braitenberg's in which we can test real neurons?

Novellino et al., (2007) and Tessadori et al., (2013) presented an experiment resembling Braitenberg's vehicles using neuron cultures. In this setup, a cart decodes distance to objects using a firing rate paradigm, and then the same paradigm is used to code back the wheels' speed independently. If the cart crashes, a stimulation of $20 \mathrm{~Hz}$ for $2 \mathrm{~s}$ is delivered (Tessadori et al., 2013). Under this protocol, the neuron culture learns to avoid obstacles. Thus, as external observers, we may be tempted to say that the cart does not like to crush, then it learns to avoid the obstacles. Even more, we are tempted to say that the goal of such behavior is to avoid crushing. However, that type of stimulation is previously known to trigger plasticity (Chiappalone et al., 2008; le Feber et al., 2010; Madhavan et al., 2007). We may also argue that each time the cart is crushed, it induced plasticity, changing the functional network. Considering how the experiment is set up, the changes will keep occurring unless crashes are avoided. Once no more crushes occur, no more changes in the network are expected. In other words, a functional neural network will keep changing until an "avoiding obstacle" structure would emerge; a moment in which we will be tempted to say that the neural culture learned to avoid obstacles. 
Critically, the functional network does not appear with by means of a goal of avoiding the impacts, rather as an effect derived from the energy demands posed by the stimulation which drives plasticity. Our proximate cause was energy demands, while the distal effect was avoiding obstacles. Importantly, this effect is structurally determined by how the wiring and stimulation conditions were set to the vehicle. Meaning that a wider set of "learnings" can emerge if the structure change. Under this framework, it is rather useless to think that at the neuron level a particular neuron or set of neurons are "processing obstacle avoidance", or that there is in the culture an obstacle avoidance network. At the level of the organism, we can be tempted to use this approach, and it might be even helpful in some contexts. Nonetheless, to explain how the vehicle learns, we must consider that individual neurons dealt with a significant energetic demand that triggers plasticity as a compensation mechanism (Vergara et al., 2019), which produces the avoidance of obstacles as emergent behavior.

It is possible to establish that in proximal terms, neurons must efficiently resolve their energy management. For example, when considering neurons' proximal context, neurons are extremely sensitive to oxygen deprivation (Ames, 2000) and the central nervous system presents small glycogen reserves (Brown and Ransom, 2007). Neurons answer to energetic demands (neural activity) outsourcing their energetic needs to the glia (Weber and Barros, 2015), which will trigger the neurovascular coupling associated with neural activity (Robinson and Jackson, 2016; Schulz et al., 2012; Sokoloff, 2008), followed by increased glucose uptake and glycolytic rate of astrocytes (Magistretti and Allaman, 2018). Not being enough, neurons' mitochondria raise ATP synthesis in response to an increment in synaptic stimuli (Connolly et al., 2014; Jekabsons and Nicholls, 2004; Lange et al., 2015; Rangaraju et al., 2014; Toloe et al., 2014). These are just the early responses in the range of hours, as the synaptic scaling ends balancing to a homeostatic level of neurons' activity 
(Barral and Reyes, 2016), reducing the energy cost of the activity increment. An increment in stimulation is expected to produce long-term network modularization (Novellino et al., 2007). Interestingly enough, when significant downscaling occurs, a few synaptic weights (dendritic spines) will increase (El-Boustani et al., 2018; Jungenitz et al., 2018). As such, while we have only described the proximal actions of neurons, they have a vast impact on the neural networks and therefore behavior. It is plausible to observe neural processing as an emergent property rooted in proximal cells requirements.

Up to this point, we have been able to rephrase neural processing without purpose or so-called "goal-oriented behaviors". Our explanation has also been faithful to a structural determinism, meaning that behavior in the vehicle emerges as a result of neurons doing the actions determined by their properties and structure. So far, introducing volition or desires in this context would be to acknowledge openly that a culture of neurons shares the same properties we usually attribute to a whole organism. However, does this reinterpretation lead to new implications?

One consequence of viewing neuronal processing as an emergent property of individual neurons displaying mechanisms that allow them to stay alive under different energetic pressures is that not everything neurons do is helpful to the organism. In other words, since neurons are only solving their local requirements, their actions may lead to the emergence of useless behaviors. This means that part of the neural network activity, which can respond to the continuous activity of multiple stimuli, will lead to the appearance of behaviors with no apparent usefulness and that areeven maladaptive. This consequence frees us from the need to include a function in every behavior we have. Many of them can be helpful for our survival and others may not, but above all, we can have neutral behaviors from an adaptative viewpoint. 
This last point is critical since the degree of behavioral flexibility increases the probability of producing neutral behaviors and deleterious ones. Thus, it is not surprising that animals with high behavioral flexibility are associated with greater effort and parenting times during ontogeny (Barton and Capellini, 2011; Heldstab et al., 2019; Isler and van Schaik, 2012; Isler and Van Schaik, 2009; Uomini et al., 2020). One only needs to observe how a toddler relates to its environment to discover that many of our behaviors during infancy put our survival or fitness at peril. Parental care or parenting allows us to buffer this flexibility, allowing us to stay alive. Conversely, flexibility also allows us to increase fitness by adapting to the environment during ontogeny, unlike less flexible animals requiring phylogenetic mechanisms of change to adapt.

\section{Building up to complex behaviors}

Behaviors flexibility by means of EHP is a powerful concept, as it allows fast changes in behavior during ontogeny, but it also allows test-retest rationale to operate. As far as test-retest rationale follows the restrictions imposed by single-cell energy management, learning can emerge. We expect that this flexibility is what is ultimately giving rise to the most complex cognitive phenomena such as understanding.

These more sophisticated cognitive phenomena are vastly discussed and modeled using the Free Energy Principle (Friston, 2010). How EHP stands in contrast to FEP? The FEP is an organisms-based approach, which considers volition as a critical element, especially when coming to aspects such as understanding (Yufik and Friston, 2016) as it distinguishes lower forms of learning, allowing the introduction of cognitive models. Therefore, as an initial difference, we noticed that FEP rather omits neuron requirements assuming them as chronically fulfilled. Secondly, it assumes the 
presence of goal-oriented behaviors, volition, and purpose, which is expected if starting from a whole organism viewpoint.

Although there are obvious differences between these two perspectives, especially since the FEP contains teleological elements and considers the nervous system as an epistemic agent (points 1 and 3 of Section 2), Yufik's proposal (Yufik, 2013, 2019; Yufik and Friston, 2016) is very similar to the EHP at the neural network level. He developed the idea of how neural assemblies (or packets) would appear, producing functional networks which allow understanding to emerge. The core idea envisions the mind as a cartographer mapping the environment, similar to classic cognitive perspectives (Bateson, 1970), where modularization of functional neural activity will allow differences to be made. In order to establish that two objects are different, a difference in the functional network should emerge (different packets or sets of packets) to allow the recognition of such distinction. Following our rationale, the critical question is the local mechanism driving the emergence of those distinctions. During the works of this thermodynamic conception of cognition, there is an acknowledgment of the relevance of energy in modulating the packets' emergence in this proposal (Yufik, 2013, 2019; Yufik and Friston, 2016). For instance, cortical tone (temperature of this thermodynamic formalization), which can be rephrased as energetic demands using EHP, is critical on how the system will react towards the equilibrium by FEP conception (Yufik, 2013). This role of energy management is even more explicit in the following communications (Yufik, 2019), mainly focused on the demand or energy expenditure and availability. Therefore, both approaches find common ground in the middle, acknowledging that local neuron requirements (i.e., energy management) are critical for modularization to occur, leading to cognitive distinctions which will ultimately produce understanding. 
It is relevant to notice that EHP and FEP are two sides of the same coin. Following the parallel conception of organism vs. cell community approach, all conceptions derived from FEP should be mapped in EHP terms and vice versa. Naturally, as we get closer to cellular processes, FEP is less precise on its implications, and when getting closer to high cognitive functions, EHP is rather vague. However, reasonable efforts can be made to understand what is happening at cellular and physiological levels when we describe the cognitive mechanism. For instance, one challenging explanation to be made from the EHP side is anticipatory behavior. How can neurons caring about their local needs resolve upcoming organism events?

One key aspect of anticipatory behavior is that it must be learned first. This means that most of the predictions we made are based upon past experiences. Therefore, we avoid pain, as we have previously experienced pain. Similar to what we described in Tessadori's vehicle case (Tessadori et al., 2013), energetic demands derived from the painful stimulation, lead to restructuration allowing pain avoidance to occur (rephrased as reducing surprise by FEP means). If we focused not on the result, but the learning phase, we would notice that consistent unrelated stimulus (e.g., a light turning on, an acoustic event, or similar signal event) is followed by pain.

Both light, sound, and pain produce energetic demands through perception. Nonetheless, the pain has a durable effect, which means a long-lasting energy demand situation. Also, its intensity is directly related to the amount of damage (Dubin and Patapoutian, 2010). Therefore, that is the critical stimulation to be avoided by means of local neuron requirements.

When we focus on neural activity during situations of these characteristics, we observe that through learning, both neural activities, the one derived from the upcoming pain signal and the one directly derived from pain, begin to fire closer in time (Urien et al., 2018). The overall activity appears to be the same, but the temporal aspects change. Basically, now the signal triggers both the signal-related 
activity and the originally pain-driven avoidance behavior. The critical aspect here is that the pain signal does not mean pain itself, but in neural activity, the signal packet (assembly) will fire just before the avoidance behavior packet. Following the logic of firing together, wired together, the avoidance packet will ultimately be activated without the pain but with the signal packet. Please note that this explanation does not require yet involve mental manipulations, and we can still be faithful to our premises.

Naturally, this explanation does not cover more sophisticated behaviors like planning which under a classic view require volitional manipulation of information. However, it sheds light on how starting from cellular communities to explain "goal-directed" behaviors can be explained leaving the goal as the consequence, not the cause. Neurons may not even realize that the animal was submitted to pain; they just react according to their local requirements. It is we, who as observers, are tempted to say that the animal learns to anticipate the aversive stimulus.

Under this context, we have given an explanation of how an organism can act in the prediction of hazard, without actually predicting it. Local neural properties allow these phenomena to occur without incorporating purpose, mental model, or further. Notably, FEP and EHP, despite their differences in starting points (and therefore conceptual frameworks), share similar predictions on how neural networks would operate. Distinctions are made on what produces those changes. Another relevant difference of our approach is that neurons can fulfill their requirements without solving the problem of the whole organism but never endangering the life of the organism (at least not immediately). Therefore, the behavioral flexibility given by the impact left by neurons when solving their needs could have a negative, neutral, or positive impact, which means that the neurons may found local energy homeostasis attractors that satisfied their requirements but not necessarily 
the organism's requirements. However, if so, why does it seem that they are almost always positive (hence the teleological need to indicate their function)?

\section{An evolutionary perspective on the coupling of different levels of operational closures.}

We see what remains, not what has been. During the evolutionary history of living beings, most species have disappeared, have become extinct (Newman, 1997). In fact, the species that are alive today represent less than $1 \%$ of the historical total (Jablonski, 2004; Newman, 1997). This makes it risky to use only evidence from modern animals to explain the relationship between cellular and whole-organism levels of organization. On the other hand, virtually all present-day animal body plans date at least back to the Cambrian explosion (CE), an event that occurred more than 500 million years ago (Maloof et al., 2010). While it is still a matter of debate, it is possible to propose that near that time window, a level of animal diversification and radiation occurred that had not been seen before and has not been seen since (Keijzer, 2015; Trestman, 2013).

Interestingly, this period has also been ascribed as when metazoans with complex active bodies appeared (Trestman, 2013). These organisms are defined by having: (i) articulated appendages; (ii) many degrees of freedom of controlled movement; (iii) true senses (such as eyes), (iv) sense-guided motility; and (v) anatomical capacity for object manipulation (Trestman, 2013). The appearance of metazoans probably occurred at least 200 million years before the CE (Dohrmann and Wörheide, 2017; Erwin, 2015), and that the nervous system probably appeared during the Ediacaran period (635 million years ago). In simpler metazoans with low-complexity nervous systems, synchrony between the neuronal and organism level was probably much easier to achieve than animals with complex active bodies. Movement is not yet a problem for those animals. Thus, it is feasible that during the initial evolution of the nervous system, a limiting element was the alignment between 
the neuronal level and that of the whole organism. Once this occurred, the space for possible radiation and diversification opened up.

In ontogenetic terms, reality is similar. In animals, the highest mortality rates are usually seen early in life (Caughley, 1966), when their individual-environment relationships are still being established. When they tend to have much more behavioral flexibility. Even in our species, this reality is not far off. For it has not been long since most of our offspring died during the first three years of life (Volk and Atkinson, 2013). The problem lies in that we often only consider its present condition when observing an organism such as ourselves. Its direct relationship with the environment, ignoring its phylogenetic and ontogenetic history. Under this perspective, most cellular phenomena are aligned with their whole organism functions. This may lead to the interpretation that the proportion of misaligned events between these levels of the organization is negligible or almost nonexistent. Thus, we only see what has worked for survival, while counterexamples of instances where cellular phenomena are misaligned with organisms vanish. In other words, under this view, we are incurring a survivorship bias, where we focus only on the instances where cellular and whole-organism levels overcame a selection process and overlook those that did not. This can lead us to false conclusions, such as overrepresenting aligned states or assuming cellular levels have functions for our survival.

This also translates into a tradeoff between flexibility and survivability. Higher degrees of freedom and higher levels of flexibility allows the emergence of novel adaptations, which increase the organism's fitness. This context can also explain why larger nervous systems (brains with more neurons) are associated with greater behavioral richness. A larger number of neurons leads to a greater diversity of local responses/solutions and greater behavioral flexibility. However, on the other hand, there must be a maximum of possible degrees of freedom before the number of misalignments between cellular and whole-organism levels can remain functional. 
Another point to consider is that not necessarily every lack of synchrony is maladaptive. There is the possibility that some of the neuronal activity that is not fully aligned with the organism is "neutral." Thus, analogous to models of neutral evolution, it is feasible that a non-trivial proportion of what neurons do to solve their local energy requirements has no significant impact on the organism's survival. It is possible to postulate that the less fundamental to survival the behavior is, the more neutral activity there is. That is, the less essential behaviors probably allow for less alignment between levels. This, in turn, would increase the presence of behavioral richness or "polymorphisms" in those behaviors.

Finally, it is critical to realize that under this notion, behaviors are not goal-oriented per se. Many may appear as goal-directed, as they are conditions of existence of the system (e.g., breathing). Under our scope, breathing organisms stay alive, therefore exist. However, breathing was never designed or deliberately addressed to solve the oxygen requirements of the organism. When we remove the goal rationale of structures and behavior, the evolutive process in which behavior emerges loses its need for teleological explanation. As such, the brain was not designed or areas within to solve specific problems. Instead, in meeting their own requirements, cells satisfy the organism's requirements too. When most cells living in the cellular community fulfill their requirement, the organisms will do so. It is simply the condition of existence of such a community. Behavioral diversity and flexibility emerge within these messy interactions of individual cells acting locally and producing distal effects that may not even affect them directly. 


\section{Final remarks}

When we observe a single cell acting in an anticipatory fashion (e.g., Shirakawa, 2006), we avoid attributing it to a sense of volition, or any epistemic or informational operation. We focus on its local mechanisms which result in such anticipatory behavior. Avoiding it is reasonable, as including it obscures the mechanisms, and also, we recognize the cell as a physical system determined by the mechanism governing it. For some reason, when coming to human beings, we failed to recognize them in such a way. This is so dramatic, that besides EHP, we have no knowledge of another integrative explicative proposal of behavior using a strict naturalistic approach.

FEP is probably the most sophisticated and flexible proposal explaining human behavior as an integrative framework. However, it uses a strong epistemic rationale to explain behavior. This leads to assigning volition to all living beings (or even dissipative systems) or stating that the concept is only applicable for certain particular systems such as human beings. Despite the differences, it is notable that the phenomena described at the neural network level, are quite similar in both proposals, meaning that both recognize more or less the same events as relevant to explain behavior. The causes of those events are different depending on which proposal framework is used.

We understand that intending to explain behavior and most sophisticated forms of it such as understanding is a major challenge for EHP. However, we consider that it is a required academic exercise in our current framework of neuroscience. As we have stated above, goals can easily emerge as observer assignation once the system is coupled with its environment, but from an evolutive perspective, adaptations don't appear to solve a problem, they just appear, and they are preserved due to advantageous impact (or at least non-deleterious). In other words, focusing on the goal may obscure the actual mechanisms which produce the phenomena we look forward to understanding. 


\section{Conflict of Interest}

The authors declare that the research was conducted in the absence of any commercial or financial relationships that could be construed as a potential conflict of interest.

\section{Author Contributions}

S.V., M.V., and R.V developed the initial general argument. All authors contributed to all the drafts; nonetheless, each author did special contributions to different sections. M.V. formalized the general systemic conditions, R.V. contribute in the neural processing and building up to behavior sections and S.V. developed the evolutionary perspective. R.V. and P.M. edited the final version.

\section{Funding}

Mario Villalobos' contribution to this paper was supported by the Agencia Nacional de Investigación

y Tecnología, ANID Chile, from a grant FONDECYT REGULAR 1194477. Pedro Maldonado's contribution to this paper was supported by Proyect ICN09_015. 


\section{References}

Ames, A. (2000). CNS energy metabolism as related to function. Brain Res. Brain Res. Rev. 34, 42-68. doi:10.1016/S0165-0173(00)00038-2.

Ashby, W. R. (1947). The nervous system as physical machine; with special reference to the origin of adaptive behaviour. Mind. 56, 44-59. doi:10.1093/mind/lvi.221.44.

Ashby, W. R. (1960). Design for a Brain. 2nd editio. London: Chapman \& Hall doi:10.2307/1418660.

Barral, J., and Reyes, A. D. (2016). Synaptic scaling rule preserves excitatory-inhibitory balance and salient neuronal network dynamics. Nat. Neurosci. 19, 1690-1696. doi:10.1038/nn.4415.

Barton, R. A., and Capellini, I. (2011). Maternal investment, life histories, and the costs of brain growth in mammals. Proc. Natl. Acad. Sci. 108, 6169-6174.

doi:10.1073/pnas.1019140108.

Bateson, G. (1970). Form, substance and difference. Essent. Readings Biosemiotics, 501.

Bechtel, W., and Richardson, R. C. (1998). "Vitalism," in Routledge Encyclopedia of Philosophy, ed. E. Craig (London: Routledge), 639-643.

Braitenberg, V. (1986). Vehicles: Experiments in Synthetic Psychology. Second. Cambridge: MIT Press.

Brown, A. M., and Ransom, B. R. (2007). Astrocyte glycogen and brain energy metabolism. Glia. doi:10.1002/glia.20557.

Caughley, G. (1966). Mortality Patterns in Mammals. Ecology 47, 906-918. doi:10.2307/1935638.

Chaffer, C. L., and Weinberg, R. A. (2011). A perspective on cancer cell metastasis. Science (80-. ). 331, 1559-1564. doi:10.1126/science.1203543.

Chiappalone, M., Massobrio, P., and Martinoia, S. (2008). Network plasticity in cortical assemblies. Eur. J. Neurosci. 28, 221-237. doi:10.1111/j.1460-9568.2008.06259.x.

Connolly, N. M. C., Dussmann, H., Anilkumar, U., Huber, H. J., and Prehn, J. H. M. (2014). Single-Cell Imaging of Bioenergetic Responses to Neuronal Excitotoxicity and Oxygen and Glucose Deprivation. J. Neurosci. doi:10.1523/JNEUROSCI.3127-13.2014.

Dada, J. O., and Mendes, P. (2011). Multi-scale modelling and simulation in systems biology. Integr. Biol. 3, 86. doi:10.1039/c0ib00075b.

DiFrisco, J. (2017). Time Scales and Levels of Organization. Erkenntnis 82, 795-818. doi:10.1007/s10670-016-9844-4.

Dohrmann, M., and Wörheide, G. (2017). Dating early animal evolution using phylogenomic data. Sci. Rep. 7, 3599. doi:10.1038/s41598-017-03791-w.

Dubin, A. E., and Patapoutian, A. (2010). Nociceptors: the sensors of the pain pathway. J. Clin. Invest. 120, 3760-3772. doi:10.1172/JCl42843.

El-Boustani, S., Ip, J. P. K., Breton-Provencher, V., Knott, G. W., Okuno, H., Bito, H., et al. (2018). Locally coordinated synaptic plasticity of visual cortex neurons in vivo. Science 
(80-. ). 360, 1349-1354. doi:10.1126/science.aao0862.

Engel, G. L. (1980). The clinical application of the biopsychosocial model. Am. J. Psychiatry 137, 535-544. doi:10.1176/ajp.137.5.535.

Erwin, D. H. (2015). Early metazoan life: divergence, environment and ecology. Philos. Trans. R. Soc. B Biol. Sci. 370, 20150036. doi:10.1098/rstb.2015.0036.

Friston, K. (2010). The free-energy principle: A unified brain theory? Nat. Rev. Neurosci. 11, 127-138. doi:10.1038/nrn2787.

Friston, K. J., and Stephan, K. E. (2007). Free-energy and the brain. Synthese 159, 417458. doi:10.1007/s11229-007-9237-y.

Heldstab, S. A., Isler, K., Burkart, J. M., and van Schaik, C. P. (2019). Allomaternal care, brains and fertility in mammals: who cares matters. Behav. Ecol. Sociobiol. 73, 71. doi:10.1007/s00265-019-2684-x.

Isler, K., and van Schaik, C. P. (2012). Allomaternal care, life history and brain size evolution in mammals. J. Hum. Evol. 63, 52-63. doi:10.1016/j.jhevol.2012.03.009.

Isler, K., and Van Schaik, C. P. (2009). Why are there so few smart mammals (but so many smart birds)? Biol. Lett. 5, 125-129. doi:10.1098/rsbl.2008.0469.

Jablonski, D. (2004). Extinction: past and present. Nature 427, 589-589.

doi:10.1038/427589a.

Jekabsons, M. B., and Nicholls, D. G. (2004). In Situ respiration and bioenergetic status of mitochondria in primary cerebellar granule neuronal cultures exposed continuously to glutamate. J. Biol. Chem. doi:10.1074/jbc.M401540200.

Jungenitz, T., Beining, M., Radic, T., Deller, T., Cuntz, H., Jedlicka, P., et al. (2018). Structural homo- and heterosynaptic plasticity in mature and adult newborn rat hippocampal granule cells. Proc. Natl. Acad. Sci. 115, E4670-E4679. doi:10.1073/pnas.1801889115.

Keijzer, F. (2015). Moving and sensing without input and output: early nervous systems and the origins of the animal sensorimotor organization. Biol. Philos. 30, 311-331. doi:10.1007/s10539-015-9483-1.

Lange, S. C., Winkler, U., Andresen, L., Byhrø, M., Waagepetersen, H. S., Hirrlinger, J., et al. (2015). Dynamic Changes in Cytosolic ATP Levels in Cultured Glutamatergic Neurons During NMDA-Induced Synaptic Activity Supported by Glucose or Lactate. Neurochem. Res. 40, 2517-2526. doi:10.1007/s11064-015-1651-9.

le Feber, J., Stegenga, J., and Rutten, W. L. C. (2010). The Effect of Slow Electrical Stimuli to Achieve Learning in Cultured Networks of Rat Cortical Neurons. PLoS One 5, e8871. doi:10.1371/journal.pone.0008871.

Madhavan, R., Chao, Z. C., and Potter, S. M. (2007). Plasticity of recurring spatiotemporal activity patterns in cortical networks. Phys. Biol. 4, 181-193. doi:10.1088/1478-

3975/4/3/005.

Magistretti, P. J., and Allaman, I. (2018). Lactate in the brain: From metabolic end-product to signalling molecule. Nat. Rev. Neurosci. doi:10.1038/nrn.2018.19. 
Maloof, A. C., Porter, S. M., Moore, J. L., Dudas, F. O., Bowring, S. A., Higgins, J. A., et al. (2010). The earliest Cambrian record of animals and ocean geochemical change. Geol. Soc. Am. Bull. 122, 1731-1774. doi:10.1130/B30346.1.

Maturana, H. (1978). "Cognition," in Wahrnehmung und Kommunikation, eds. P. M. Hejl, W. K. Köck, and G. Roth (Frankfurt: Peter Lang), 29-49. doi:10.1007/978-3-662-493250_15.

Maturana, H. (2002). Autopoiesis, Structural Coupling and Cognition: A history of these and other notions in the biology of cognition. Cybern. Hum. Knowing 9, 5-34.

Maturana, H. R. (2008). Anticipation and Self-consciousness. Are these Functions of the Brain? Constr. Found. 4, 18-20.

Maturana, H. R., and Varela, F. J. (1980). "Problems in the Neurophysiology of Cognition," in, 41-47. doi:10.1007/978-94-009-8947-4_5.

Maturana, H. R., and Varela, F. J. (1989). De boom der kennis. Hoe wij wereld door onze Eig. Waarn. creëren, 58-60.

Mayr, E. (1961). Cause and Effect in Biology Linked references are available on JSTOR for this article : Cause and Effect in Biology Kinds of causes, predictability, and teleology. Science (80-. ). 134, 1501-1506. Available at:

http://www.jstor.org.ezproxy.auckland.ac.nz/stable/1707986.

McGregor, S., and Virgo, N. (2011). Life and its close relatives. Lect. Notes Comput. Sci. (including Subser. Lect. Notes Artif. Intell. Lect. Notes Bioinformatics) 5778 LNAI, 230237. doi:10.1007/978-3-642-21314-4_29.

Newman, M. E. J. (1997). A Model of Mass Extinction. J. Theor. Biol. 189, 235-252. doi:10.1006/jtbi.1997.0508.

Novellino, A., D’Angelo, P., Cozzi, L., Chiappalone, M., Sanguineti, V., and Martinoia, S. (2007). Connecting Neurons to a Mobile Robot: An In Vitro Bidirectional Neural Interface. Comput. Intell. Neurosci. 2007, 1-13. doi:10.1155/2007/12725.

Park, C. O., and Kupper, T. S. (2015). The emerging role of resident memory T cells in protective immunity and inflammatory disease. Nat. Med. 21, 688-697. doi:10.1038/nm.3883.

Pedersen, S. F., Kapus, A., and Hoffmann, E. K. (2011). Osmosensory mechanisms in cellular and systemic volume regulation. J. Am. Soc. Nephrol. 22, 1587-1597.

doi:10.1681/ASN.2010121284.

Rangaraju, V., Calloway, N., and Ryan, T. A. (2014). Activity-driven local ATP synthesis is required for synaptic function. Cell. doi:10.1016/j.cell.2013.12.042.

Robinson, M. B., and Jackson, J. G. (2016). Astroglial glutamate transporters coordinate excitatory signaling and brain energetics. Neurochem. Int. 98, 56-71.

doi:10.1016/j.neuint.2016.03.014.

Roux, E. (2014). The concept of function in modern physiology. J. Physiol. 592, 22452249. doi:10.1113/jphysiol.2014.272062.

Schulz, K., Sydekum, E., Krueppel, R., Engelbrecht, C. J., Schlegel, F., Schröter, A., et al. (2012). Simultaneous BOLD fMRI and fiber-optic calcium recording in rat neocortex. Nat. 
Methods 9, 597-602. doi:10.1038/nmeth.2013.

Shirakawa, T. (2006). Anticipatory Behavior and Intracellular Communication in Physarum polycephalum. in AIP Conference Proceedings (AIP), 541-546. doi:10.1063/1.2216665.

Sokoloff, L. (2008). The physiological and biochemical bases of functional brain imaging. Cogn. Neurodyn. doi:10.1007/s11571-007-9033-x.

Southern, J., Pitt-Francis, J., Whiteley, J., Stokeley, D., Kobashi, H., Nobes, R., et al. (2008). Multi-scale computational modelling in biology and physiology. Prog. Biophys. Mol. Biol. 96, 60-89. doi:10.1016/j.pbiomolbio.2007.07.019.

Tessadori, J., Venuta, D., Kumar, S. S., Bisio, M., Pasquale, V., and Chiappalone, M. (2013). Embodied neuronal assemblies: A closed-loop environment for coding and decoding studies. Int. IEEE/EMBS Conf. Neural Eng. NER, 899-902.

doi:10.1109/NER.2013.6696080.

Toloe, J., Mollajew, R., Kügler, S., and Mironov, S. L. (2014). Metabolic differences in hippocampal "Rett" neurons revealed by ATP imaging. Mol. Cell. Neurosci.

doi:10.1016/j.mcn.2013.12.008.

Trestman, M. (2013). The Cambrian Explosion and the Origins of Embodied Cognition. Biol. Theory 8, 80-92. doi:10.1007/s13752-013-0102-6.

Ulanowicz, R. E., and Hannon, B. M. (1987). Life and the production of entropy. Proc. $R$. Soc. London. Ser. B. Biol. Sci. 232, 181-192. doi:10.1098/rspb.1987.0067.

Uomini, N., Fairlie, J., Gray, R. D., and Griesser, M. (2020). Extended parenting and the evolution of cognition. Philos. Trans. R. Soc. B Biol. Sci. 375, 20190495.

doi:10.1098/rstb.2019.0495.

Vergara, R. C., Jaramillo-Riveri, S., Luarte, A., Moënne-Loccoz, C., Fuentes, R., Couve, A., et al. (2019). The Energy Homeostasis Principle: Neuronal Energy Regulation Drives Local Network Dynamics Generating Behavior. Front. Comput. Neurosci. 13. doi:10.3389/fncom.2019.00049.

Villalobos, M. (2015). The Biological Roots of Cognition and the Social Origins of Mind.

Villalobos, M., and Ward, D. (2015). Living Systems: Autonomy, Autopoiesis and Enaction. Philos. Technol. 28, 225-239. doi:10.1007/s13347-014-0154-y.

Volk, A. A., and Atkinson, J. A. (2013). Infant and child death in the human environment of evolutionary adaptation. Evol. Hum. Behav. 34, 182-192.

doi:10.1016/j.evolhumbehav.2012.11.007.

Weber, B., and Barros, L. F. (2015). The Astrocyte: Powerhouse and Recycling Center. Cold Spring Harb. Perspect. Biol., a020396. doi:10.1101/cshperspect.a020396.

Yufik, Y. (2013). Understanding, consciousness and thermodynamics of cognition. Chaos, Solitons \& Fractals 55, 44-59. doi:10.1016/j.chaos.2013.04.010.

Yufik, Y. (2019). The Understanding Capacity and Information Dynamics in the Human Brain. Entropy 21, 308. doi:10.3390/e21030308.

Yufik, Y., and Friston, K. (2016). Life and Understanding: The Origins of "Understanding" in Self-Organizing Nervous Systems. Front. Syst. Neurosci. 10, 1-23. 
doi:10.3389/fnsys.2016.00098. 